\title{
GERENCIAMENTO HÍBRIDO DE PROJETOS PARA PRODUTOS FÍSICOS - UMA ANÁLISE BIBLIOMÉTRICA E SISTÊMICA
}

\author{
[ Autor 1 ] Ana Rita Villela Costa (anaritavc@uol.com.brl) - PPGEM, Universidade Tecnológica \\ Federal do Paraná
}

[ Autor 2 ] Milton Borsato (borsato@utfpr.edu.br) - PPGEM, Universidade Tecnológica Federal do Paraná

[ Autor 3 ] Gisele Bortolaz (gisele @ bortolaz.com.br) - PPGEM, Universidade Tecnológica Federal do Paraná

[ Autor 4 ] Jaqueline Sebastiany Iaksch (jaqsiasksch@ gmail.com) - PPGEM, Universidade Tecnológica Federal do Paraná

\section{RESUMO}

Um dos fatores de sucesso das organizações é o rápido lançamento dos seus produtos no mercado. Partindo desse pressuposto, o Gerenciamento Ágil de projetos surgiu para diminuir o tempo entre a ideia e o lançamento do produto. Vários estudos vêm sendo desenvolvidos para unir as qualidades do Gerenciamento Ágil com o Gerenciamento de Projetos tradicional para aplicação no desenvolvimento de produtos físicos convencionais, tais como tratores e automóveis. Destes estudos surge a questão: é possível aplicar o Gerenciamento Híbrido (i.e. tradicional e ágil) para este tipo de produto fisico? $O$ presente trabalho busca responder essa pergunta, através de uma análise bibliométrica e sistêmica baseada no método ProKnow-C (Knowledge Development Process - Constructivist). Inicialmente foi realizada a etapa de seleção do portfólio bibliográfico representativo do tema de pesquisa. Nesta etapa foram definidos os eixos de pesquisa, as palavras-chave, assim como foram realizadas buscas em bases de dados e filtragens de artigos. Desta forma, foi construído um portfólio bibliográfico composto de 39 artigos alinhados ao tema de pesquisa. Posteriormente foi realizada a etapa de análise bibliométrica, na qual identificou-se a relevância dos periódicos, a relevância dos autores, as principais palavraschave e a evolução do tema de pesquisa nos últimos 5 anos. Finalmente, na etapa de análise sistêmica, foi possível avaliar o conteúdo dos artigos do portfólio a fim de identificar as principais tendências e oportunidades de pesquisa, dentre as quaispode-se destacar: (i) estudo comparativo entre equipes colocalizadas e à distância; (ii) aplicação do modelo híbrido num projeto piloto; (iii) investigar o principal fator de sucesso da Metodologia Ágil aplicada a um modelo híbrido; (iv) participação do cliente ativa do cliente durante os "sprints" identificando os requisitos do produto.

Palavras chave: Gerenciamento Ágil; Desenvolvimento de Produto; Bibliometria; Análise Sistêmica Área: Gestão do Processo de Desenvolvimento de Produtos e Serviços.

\section{INTRODUÇÃO}

Com o avanço da concorrência e da tecnologia, é de fundamental importância para o sucesso de uma companhia, que seus produtos sejam lançados no mercado o mais rápido possível. Em estudos recentes concluiu-se que apenas uma pequena parcela dos novos produtos são lançados a tempo (em média 44\%) e que apenas a metade dos novos produtos geram lucro para as empresas (COOPER, 2014). Por estas razões é de suma importância que o desenvolvimento de novos produtos seja uma prioridade para as empresas e que boas práticas sejam aplicadas para 
melhorar cada vez mais esse processo.

A indústria vem enfrentando grandes desafios devido ao aumento da variedade de produtos, à inconstância do mercado e à fabricação distribuída. Uma consequência da globalização e da volatilidade do mercado é a crescente complexidade dos sistemas econômico, fabril e tecnológico. Assim, baseado no Manifesto Ágil de 2001, que é um conjunto de guias e regras de como desenvolver novos softwares (COOPER; SOMMER, 2016), surgiu a Metodologia Ágil.

O sistema de Gerenciamento Ágil funciona muito bem na área de TI, porém para utilizá-lo em desenvolvimento de produtos físicos possui alguns inconvenientes. A Metodologia Ágil utiliza o desenvolvimento em espiral que baseia-se em "construir-testar-retornar-revisar" o produto nos vários estágios de desenvolvimento, contando com a participação ativa do cliente. (COOPER, 2014). A Gestão Ágil diminui o tempo entre a ideia e o lançamento dos novos produtos pois as etapas se sobrepõem, começando uma etapa sem antes ter terminado a anterior. Segundo Cooper (2014), o processo ocorre como no futebol americano (Scrum) com atividades múltiplas e paralelas como numa corrida.

Segundo Carulli, Bordegoni e Cugini (2013) a participação da voz do cliente têm sido utilizada para a customização de produtos como sapatos, roupas entre outros. As pesquisas para determinar os desejos dos clientes vêm sendo desenvolvidas pelas equipes de marketing das companhias porém, na transferência das informações para o desenvolvimento do produto aparecem distorções. A tendência atual é de envolver, cada vez mais, os engenheiros e projetistas na captação das necessidades dos clientes para que estas sejam convertidas em requerimentos do produto, gerando uma maior satisfação dos clientes.

Dessa maneira, esse artigo tem como objetivo identificar as soluções propostas e as tendências acerca do estudo sobre Gerenciamento Ágil e Híbrido, através da revisão da literatura com base em estudos realizados nos últimos cinco anos. Para alcançar esse objetivo, o presente estudo baseou-se no método ProKnow-C (Knowledge Development Process - Constructivist).

$\mathrm{Na}$ Seção 2 são apresentados os aspectos metodológicos da pesquisa, seguidos da apresentação da sequência de atividades realizadas para a seleção do portfólio bibliográfico (Seção 3). A análise bibliométrica e a análise sistêmica são apresentadas nas Seções 4 e 5, respectivamente. Por fim, na Seção 6, são apresentadas as conclusões.

\section{ASPECTOS METODOLÓGICOS}

Segundo Prodanov e Freitas (2013), a pesquisa científica tem o objetivo de conhecer e explicar os fenômenos e, para tanto, utiliza técnicas diversas para obter os resultados desejados. Quanto aos objetivos, as pesquisas podem ser classificadas como: exploratória, explicativa e descritiva. Quanto aos procedimentos metodológicos são classificadas como: documental, bibliográfica, experimental, operacional, estudo de caso, entre outas (PRODANOV; DE FREITAS, 2013). Portanto, quanto ao objetivo esta pesquisa é do tipo exploratória, pois busca investigar o assunto de forma preliminar com o objetivo de delimitar o tema de pesquisa, assim como encontrar as tendências futuras acerca do assunto explorado. O procedimento adotado é a pesquisa bibliográfica e escolheu-se o Knowledge Development Process-Constructivist (Proknow-C) como ferramenta de pesquisa.

O ProKnow-C tem o objetivo de identificar artigos acadêmicos com forte relevância à pesquisa. Esse método é composto basicamente de três passos: seleção do portfólio, análise bibliométrica e análise sistêmica do portfólio (TASCA et al., 2010). Desta maneira, é possível definir as perguntas e as oportunidades de pesquisa relativas a um tema específico. Na etapa de definição do portfólio bibliográfico, são definidos os eixos de pesquisa, as palavras-chave e as bases de 
dados, para na sequência realizar a busca de artigos nas bases disponíveis no Portal de Periódicos da CAPES. Através do resultado desta busca, inicia-se a etapa de análise bibliométrica, na qual são definidos os autores, os periódicos e as palavras-chave mais relevantes, assim como a evolução do tema de pesquisa. A terceira etapa, denominada análise sistêmica, consiste na análise do conteúdo dos artigos do portfólio bibliográfico quanto aos seus objetivos, a metodologia, aos resultados, entre outros parâmetros.

Com o presente estudo, pretende-se definir as oportunidades e tendências de pesquisa, bem como o estado da arte do tema. Para apoiar o desenvolvimento desta pesquisa, utilizou-se o programa EndNote para o gerenciamento da bibliografia e o Microsoft Excel para compilação de dados, elaboração dos gráficos e análises.

\section{SELEÇÃO DO PORTFÓLIO BIBLIOGRÁFICO}

Para a seleção do Portfólio Bibliográfico, inicialmente foi realizada a seleção da base de artigos brutos e na sequência, esta base foi filtrada com base em alguns parâmetros predefinidos.

\subsection{Seleção da base de artigos brutos}

Para a seleção dos artigos brutos as seguintes etapas foram seguidas: 1- Definição dos eixos e palavras-chave; 2- Seleção da base de artigos; 3- Busca nas bases de artigos 4- Definição dos filtros do portfólio e 5- Formação do banco de artigos bruto. As etapas serão detalhadas a seguir.

\subsubsection{Definição dos eixos e palavras-chave}

Os eixos de pesquisa foram definidos de acordo com o objetivo do presente trabalho, sendo estes: i) Agile Product Management e ii) New Product Development. As palavras-chave foram definidas, após a leitura de alguns artigos mais recentes acerca do assunto tratado. Como a grande parte dos artigos publicados estão na língua inglesa optou-se pelo uso de palavras-chave em inglês. Na tabela 1 estão os eixos e as respectivas palavras-chave.

Tabela 1. Eixos de pesquisa e palavras-chave. Fonte: A autora.

\begin{tabular}{|l|l|}
\hline \multicolumn{1}{|c|}{ Eixos } & \multicolumn{1}{|c|}{ Palavras Chaves } \\
\hline New Product Development & New Product Development \\
& NPD \\
& Product Development \\
& Product Development Process \\
\hline Agile Product Management & Agile Development \\
& scrum \\
& Burndown charts \\
& Project Management \\
& Accelerated development \\
Adaptive development
\end{tabular}

\subsubsection{Seleção das Bases de Artigos}

As bases de artigos foram selecionadas de acordo com as áreas de interesse no Portal de Periódicos da CAPES (Coordenação de Aperfeiçoamento de Pessoal de Nível Superior). Assim, as bases escolhidas foram: i) Emerald; ii) Engineering Village (COMPEDEX); iii) IEEE; iv) Wiley; v) ProQuest; vi) Scopus; vii) Science Direct; viii) Springer Link; ix) Web of Science (Scielo) e x) Web of Science. Quanto à representatividade das mesmas, ficou definido que se a base retornasse pelo menos um artigo, esta seria mantida. Dessa maneira, todas as bases foram mantidas no processo. 


\subsubsection{Busca nas Bases de Artigos}

Após a definição das bases, realizou-se a busca em cada uma delas utilizando os operadores booleanos "AND" e "OR", sendo o operador "AND" empregado para unir os eixos e o "OR" para unir as palavras-chave.Assim, as buscas foram realizadas com o seguinte truncamento de palavras: ("New Product Development" OR "NPD" OR "Product Development" OR "Product Development Process") AND ("Agile Development" OR "scrum” OR "Burndown charts" OR "Project Management" OR “Accelerated development" OR “Adaptive development”).

\subsubsection{Definição dos Filtros do Portfólio}

Para aplicar um filtro nas bases de dados foram considerados apenas artigos de periódicos e de congressos, no período entre 2012 e 2017.

\subsubsection{Formação do Banco de Artigos Bruto}

Como resultado das etapas anteriores, formou-se um banco de artigos bruto composto por 1.536 artigos. As buscas foram realizadas entre os dias 06 e 09 de março de 2017.

\subsection{Filtragem do Banco de Artigos}

Todas as referências do banco de artigos bruto foram exportadas para o Software de gerenciamento bibliográfico EndNote, no qual realizou-se as etapas de filtragem. Inicialmente, foram eliminados os artigos em duplicidade e os artigos de capítulos e revistas, restando assim 1.363 artigos. Na sequência, foram excluídos 358 artigos, por estarem atrelados a outras áreas de conhecimento, apesar do filtro da seleção. Nos 1005 artigos restantes, foi realizada a leitura dos títulos a fim de verificar a aderência com o tema. Desta maneira, identificou-se que 145 artigos estavam aderentes ao tema de pesquisa. A seguir, foram lidos os resumos desses 145 artigos e apenas 63 foram considerados alinhados com o tema da pesquisa.

$\mathrm{Na}$ etapa subsequente, os artigos foram analisados quanto ao reconhecimento científico através da busca de citações de cada um no Google Scholar. O critério de relevância adotado foi a existência de pelo menos uma citação em cada artigo. Os artigos sem citação foram retirados do portfólio principal para serem analisados posteriormente e o portfólio provisório ficou composto por 38 artigos.

Na sequência, foram identificados os principais autores do tema da pesquisa com o objetivo de resgatar artigos que foram eliminados, mas que poderiam ser relevantes por serem escritos por estes autores. Desta maneira, retornou-se ao portfólio de artigos com títulos alinhados para identificar artigos escritos pelos autores principais, porém nenhum artigo foi encontrado nessa nova avaliação. Por fim, os artigos que não possuíam pelo menos uma citação foram reavaliados. Um destes artigos, publicado no ano de 2016, foi realocado ao portfólio provisório. Assim, o portfólio bibliográfico final, representativo do tema de pesquisa abordado, passou a ser composto por 39 artigos. A definição do portfólio final ocorreu em 18 de Abril de 2017. A figura 1 ilustra o procedimento detalhado nesta seção. 
Figura 1. Seleção do portfólio bibliométrico. Fonte: A autora.

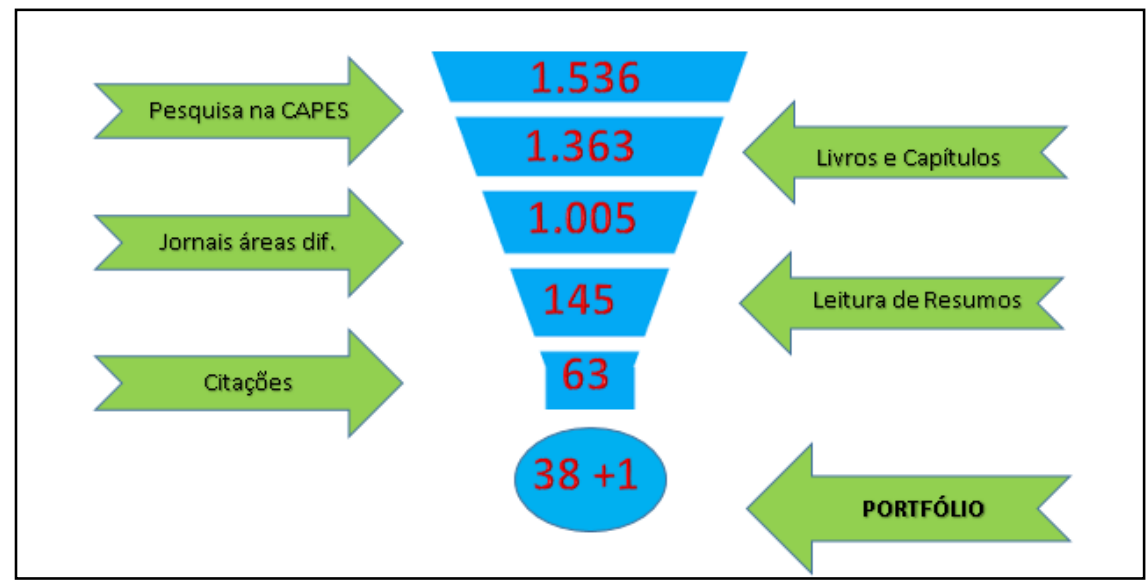

\section{ANÁLISE BIBLIOMÉTRICA DO PORTFÓLIO}

A análise bibliométrica tem o objetivo de avaliar e interpretar o portfólio bibliográfico. Com a realização desta análise, pode-se identificar os autores que mais se destacam na área de pesquisa, o ano de publicação dos artigos, as palavras-chave encontradas no portfólio, bem como a relevância dos periódicos e congressos acerca do tema de pesquisa.

A primeira análise realizada é relacionada aos autores. Os 39 artigos, que compõem o portfólio bibliográfico, foram desenvolvidos por um total de 106 autores. Destes autores, identifica-se a presença de alguns pesquisadores brasileiros, os quais possuem mais de um artigo publicado (E.C. Conforto e D.C. Amaral - ambos com dois artigos publicados cada). A autora A. F. Sommer publicou a maior quantidade de artigos relativos ao tema, com cinco publicações. $\mathrm{O}$ autor R. G. Cooper publicou três artigos, já o autor I. Dukovska-Popovska publicou dois. Os demais autores publicaram apenas um artigo cada.

A segunda análise está relacionada ao ano de publicação dos artigos. Observou-se que a maioria dos artigos foram publicados em 2012 e em 2016, sendo que um artigo de 2017 aparece no portfólio (Figura 2).

Figura 2. Artigos publicados por ano. Fonte: A autora.

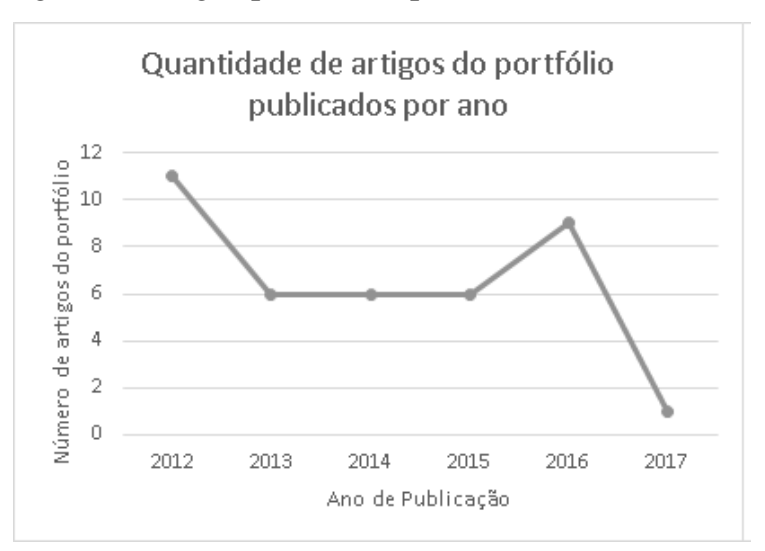

Em uma terceira análise, foram verificadas as palavras-chave encontradas nos artigos do portfólio. Fez-se o agrupamento dos termos semelhantes, resultando num total de 189 palavras. 
Pode-se perceber que 123 palavras apareceram apenas uma vez nos artigos. A figura 3 apresenta as palavras-chave mais utilizadas no portfólio bibliográfico, na qual destacam-se as palavras Product Development, Project Management e Software foram identificadas em 9 artigos do portfólio bibliográfico. As palavras-chave Agile Software Development e Scrum apareceram em 5 artigos. Entende-se, assim, que as palavras definidas no início desta pesquisa estavam alinhadas aos resultados encontrados.

Figura 3. Aderência das palavras-chave. Fonte: A autora.

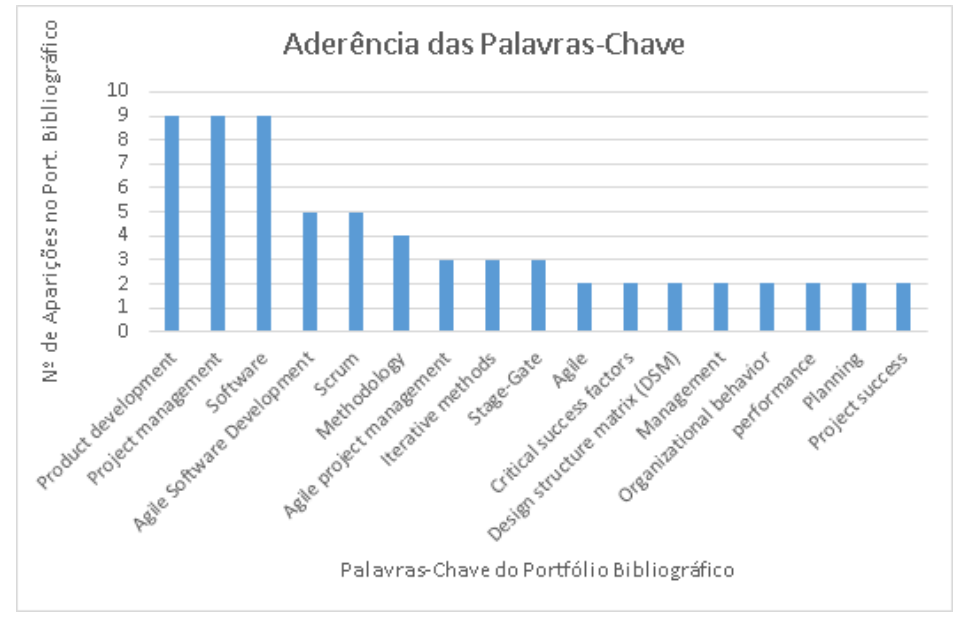

Em uma quarta e última análise, os periódicos do portfólio bibliográfico foram organizados por grau de relevância. Pode-se verificar, que o periódico Information and Software Technology é o mais representativo no portfólio, seguido respectivamente do International Journal of Project Management e IEEE Transactions on Engineering Management (Figura 4).

Figura 4. Relevância dos periódicos no portfólio bibliométrico. Fonte: A autora.

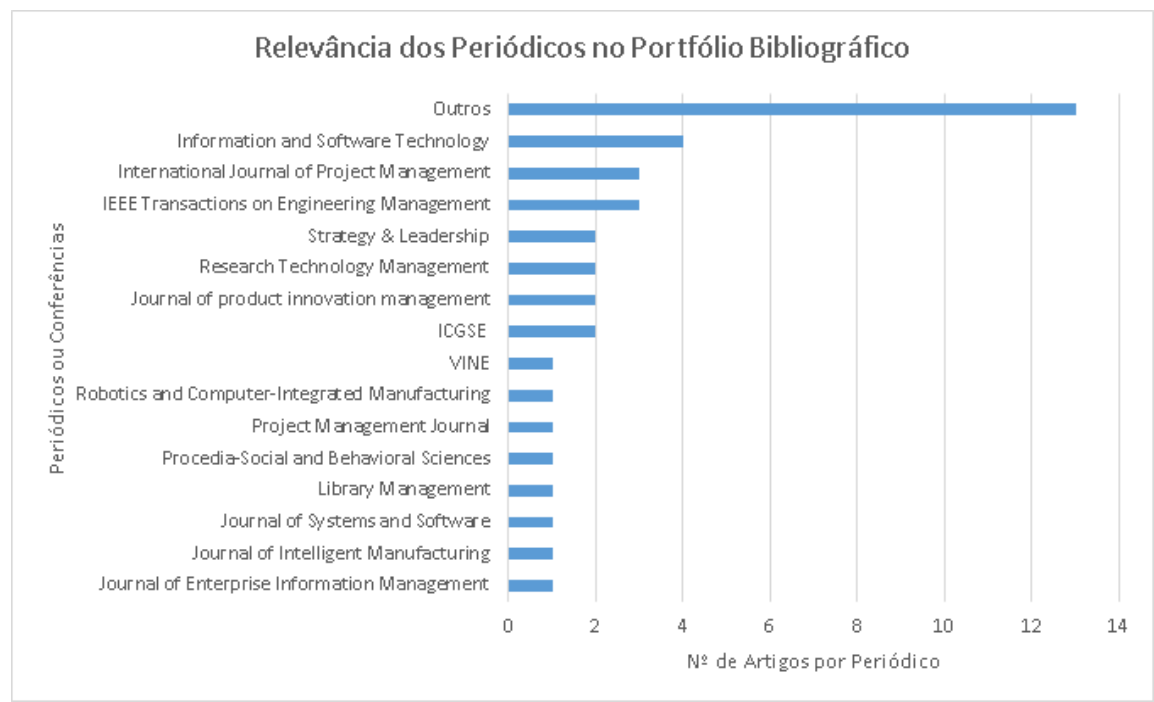

Após a etapa de análise bibliométrica, partiu-se para o desenvolvimento da análise sistêmica, a qual será apresentada na próxima Seção. 


\section{ANÁLISE SISTÊMICA}

A análise sistêmica tem o objetivo de analisar uma amostra representativa de um dado assunto do portfólio bibliográfico (ENSSLIN et al., 2010). Para a realização desta análise todos os artigos do portfólio foram lidos na íntegra e alguns aspectos foram tabulados para se identificar as oportunidades e tendências almejadas. Os seguintes aspectos foram tabulados: i) Objetivos; ii) Tipo de Artigo; iii) Unidade de Análise; iv) Metodologia; v) Resultados; vi) Recomendações Futuras e vii) Oportunidades. Assim foram identificadas as oportunidades de pesquisa detalhadas a seguir.

\subsection{Tendências e oportunidades de pesquisa}

As oportunidades encontradas estão divididas em dois grupos, sendo o primeiro na área de Gerenciamento Ágil de Projetos e a segunda na área de Desenvolvimento Híbrido de Projetos.

\subsubsection{Gerenciamento Ágil de Projetos}

a) Estudo comparativo entre equipes colocalizadas e à distância

Os estudos de caso realizados por Hadar et al. (2013) em empresas de software mostraram-se eficientes na redução de documentação de projeto. Por outro lado, os estudos de Moe, Aurum e Dybå (2012) e de Drury-Grogan (2014), apresentaram eficiência em compartilhar o conhecimento com os times e em ter equipes auto-organizadas e colocalizadas. Uma das premissas do Gerenciamento Ágil é que o time seja auto-organizado e colocalizado porém quatro autores, Sungkur e Ramasawmy (2014) e Ieris e Salinger (2013) propõem soluções para times a distância, já que ter empresas globalizadas é uma tendência mundial. Assim, identificase como oportunidade de pesquisa a realização de um estudo comparativo da aplicação da Metodologia Ágil com times colocalizados e com times globalizados.

\subsubsection{Desenvolvimento Híbrido de Projetos}

Neste grupo as oportunidades de pesquisa podem ser divididas em três subgrupos, conforme descritos a seguir.

\section{a) Aplicação do modelo híbrido num projeto piloto}

A maioria das pesquisas na utilização da Metodologia Ágil em conjunto com um modelo "tradicional" de desenvolvimento de produto físicos são estudos de caso fornecendo resultados empíricos (COOPER; SOMMER, 2016), (SOMMER et al., 2014), (CONFORTO; AMARAL, 2016); (JHA; VILARDELL; NARAYAN, 2016), (COOPER; SOMMER, 2016); (SOMMER et al., 2015) e (EKLUND; BOSCH, 2012). Para aumentar a confiabilidade dos resultados esses autores sugerem o aumento das amostras nos estudos futuros, bem como a aplicação de um modelo híbrido numa maior diversidade de produtos para obter uma base de comparação. Neste sentido uma das oportunidades de pesquisa encontrada é a aplicação do modelo híbrido em um projeto piloto (com adaptações) para avaliação da sua eficácia.

b) Fator de sucesso da Metodologia Ágil

Nesta área alguns resultados apresentados foram qualitativos e não ficou muito claro qual ou quais aspectos da Metodologia Ágil são responsáveis pelo sucesso do projeto. Uma oportunidade de pesquisa é investigar, num modelo aplicado, qual o principal fator de sucesso da Metodologia Ágil aplicada a um modelo híbrido. 
c) Participação do cliente

O Gerenciamento Ágil propõe a participação ativa do cliente durante os "sprints" identificando os requisitos do produto. Para o desenvolvimento de software, o produto funcional produzido é composto por linhas de programa e facilita a construção de vários "protótipos" durante os diversos "sprints". Para os produtos físicos, o tempo e custo requerido para a produção de produtos funcionais é bem maior e mais complexo. Segundo Carulli, Bordegoni e Cugini (2013) a aplicação de um protótipo virtual, em que o usuário pode interagir com o produto através de uma plataforma virtual, diminui o tempo e custo da criação do protótipo podendo assim utilizálo nos "sprints" do modelo híbrido para produtos físicos. Desta maneira, o protótipo virtual viabiliza a participação do cliente no projeto. Neste sentido, uma oportunidade de pesquisa é desenvolver um protótipo virtual para um produto físico para verificar a eficácia no Gerenciamento Híbrido de Projetos.

\section{CONCLUSÃO}

O presente estudo identificou algumas oportunidades de pesquisas futuras e as tendências do tema. Através desse levantamento verificou-se a necessidade de executar estudos que apliquem a Metodologia Ágil em times colocalizados e globalizados, além de estudos melhor estruturados, de maneira a fornecer resultados cientificamente mais confiáveis. Sugere-se também após essa análise, o desenvolvimento de um protótipo virtual para um produto físico, tornando-se possível verificar a real eficácia no Gerenciamento Híbrido de Projetos. Da mesma forma, identificar o principal fator de sucesso da Metodologia Ágil aplicada a um modelo híbrido mostra-se como sendo uma oportunidade de pesquisa bastante relevante ao tema desse estudo.

Com o avanço da tecnologia e a velocidade de lançamento de produtos num mundo cada vez mais globalizado, o uso de métodos e modelos para diminuir o time to Market é uma das principais tendências identificadas neste trabalho.

Como trabalho futuro, recomenda-se realizar o procedimento novamente, com o intuito de atualizar o portfólio e levantar novas oportunidades e tendências relacionados ao tema.

\section{REFERÊNCIAS}

CARULLI, M.; BORDEGONI, M.; CUGINI, U. An approach for capturing the Voice of the Customer based on Virtual Prototyping. Journal of Intelligent Manufacturing. v24, n.5, p.887-903, 2013.

CONFORTO, E. C.; AMARAL, D. C. Agile project management and stage-gate model-A hybrid framework for technology-based companies. Journal of Engineering and

Technology Management, v40, p.1-14, 2016.

COOPER, R. G. What's next? After stage-gate. Research Technology Management, v57, n.1, p.20-31, 2014.

COOPER, R. G.; SOMMER, A. F. The AgileStage-Gate Hybrid Model: A Promising New Approach and a New Research Opportunity. Journal of Product Innovation Management, v33, n.5, p.513-526, 2016. 
DRURY-GROGAN, M. L. Performance on agile teams: Relating iteration objectives and critical decisions to project management success factors. Information and Software Technology, v56, n.5, p.506-515, 2014.

EDUARDO TASCA, J.; ENSSLIN, L.; ROLIM ENSSLIN, S.; BERNARDETE MARTINS ALVES, M. An approach for selecting a theoretical framework for the evaluation of training programs. Journal of European Industrial Training, v34, n.7, p.631-655, 2010.

EKLUND, U.; BOSCH, J. Applying agile development in mass-produced embedded systems. In: 13th International Conference on Agile Software Development. Malmo, Sweden:Springer Verlag, p.31-46, 2012.

ENSSLIN, L.; ENSSLIN, S. R.; LACERDA, R. T. D. O.; TASCA, J. E. ProKnow-C, knowledge development process-constructivist. Processo técnico com patente de registro pendente junto ao INPI. Brasil, v10, n.4, p.2015, 2010.

HADAR, I.; SHERMAN, S.; HADAR, E.; HARRISON, J. J. Less is More: Architecture Documentation for Agile Development. 6th International Workshop on Cooperative and Human Aspects of Software Engineering. p.121-124, 2013.

JHA, M. M.; VILARDELL, R. M. F.; NARAYAN, J. Scaling agile scrum software development: Providing agility and quality to platform development by reducing time to market. In: 11th IEEE International Conference on Global Software Engineering, ICGSE, p. 84-88, 2016.

KEVIN SUNGKUR, R.; RAMASAWMY, M. Knowledge4Scrum, a novel knowledge management tool for agile distributed teams. VINE, v44, n.3, p.394-419, 2014.

MOE, N. B.; AURUM, A.; DYBÅ, T. Challenges of shared decision-making: A multiple case study of agile software development. Information and Software Technology, v54, n.8, p.853-865, 2012.

PRODANOV, C. C.; DE FREITAS, E. C. Metodologia do Trabalho Científico: Métodos e Técnicas da Pesquisa e do Trabalho Acadêmico. $2^{\text {a }}$ Ed: Editora Feevale, 2013

SOMMER, A. F.; SLAVENSKY, A.; THUY NGUYEN, V.; STEGER-JENSEN, K.; DUKOVSKA-POPOVSKA, I. Scrum integration in stage-gate models for collaborative product development - A case study of three industrial manufacturers. In: 2013 IEEE International Conference on Industrial Engineering and Engineering Management. p. 1278-1282, 2013.

SOMMER, A. F.; HEDEGAARD, C.; DUKOVSKA-POPOVSKA, I.; STEGER-JENSEN, K. Improved product development performance through agile/stage-gate hybrids: The nextgeneration stage-gate process? Research Technology Management, v58, n.1, p.34-44, 2015. ZIERIS, F.; SALINGER, S. Doing Scrum Rather Than Being Agile: A Case Study on Actual Nearshoring Practices. IEEE 8th International Conference on Global Software Engineering (Icgse 2013). p. 144-153, 2013. 